\title{
COMPOSTOS BIOLOGICAMENTE ATIVOS EM INVERTEBRADOS MARINHOS
}

\author{
JOSÉ CARLOS DE FREITAS \\ Departamento de Fisiologia Geral - Instituto de Biociências \\ Universidade de São Paulo, São Paulo, SP, Brasil
}

\section{SYNOPSIS}

The author has reviewed the classification, the nomenclature and the ecological aspects of biologically active compounds, emphasizing the Freyvogel \& Perret's (1973) concepts of venomous and poisonous organisms. The literature involving the phyla Annelida, Mollusca and Arthropoda was also reviewed. The author's pharmacological studies performed with the polychaete Eurythoe camplanata, which revealed a dializable principle, active in the guinea pig's ileum and dorsal muscle ot polvchaete itselJ are also commented. This principle differs from A cetylcholine and Serotonin. Pharmacologically studies in the sea-hares (genus Aplysia) were also focused, including the author's contribution. In the glands os Aplysia brasiliana, namely the mid-gut-gland, the opaline gland and the purple gland, the author confirmed presence of cholinesters, which are discussed in the review. Toxicity of Arthropods are also discussed, mainly in decapod crustaceans. In some Brazilian crabs studies revealed a pharmacological activity of the gastric secretion expelled with an apparently defensive purpose and its ecological importance is focused in terms of the community. The relatively scarce studies of chemical ecology of marine organisms in Latin America and the impor. tance of these studies were finally emphasized.

\section{Introdução}

Em todos os niveis da escola filogenética, encontramos exemplos de ataque, defesa e resposta comportamental bảseada em agentes químicos e as interações e caracterizações dos agentes químicos envolvidos constituem objeto de estudo da ecologia química (Sondheimer \& Simeone, 1970; Kittredge et al., 1974). Os produtos químicos sintetizados pelos organismos e que servem à interação química entre os membros de uma mesma espécie, constituem os Feromônios (Karlson \& Luscher, 1959), dos quais são exemplos marinhos o feromônio de alarme do Diadema antillarum (Echinodermata, Echinoidea), que é liberado quando um membro do grupo é lesado, desencadeando o afastamento dos companheiros (Mackie \& Grant, 1974), e o da anêmona colonial Anthopleura elegantíssima, onde se nota a contração dos outros membros da "colonia" em resposta a uma substância, liberada por um indivíduo lesado, que foi denominada Antopleurina (Howe \& Sheikh, 1975). O fenômeno da "epidemic spawning" é verificado num outro equinoide, Paracentrotus lividus, em que a água contendo gametas induz os outros membros a liberarem seus produtos genitais (Fox, 1974).

Os produtos químicos que medeiam interaç̃es entre indivíduos de espécies diferentes (excluindo substâncias usadas como alimento), pertencem à categoria dos Aleloquímicos, quando conferem vantagens adaptativas aos organismos liberadores são denominados Alomônios, enquanto que aqueles que dão vantagens adaptativas aos organismos receptores denominam-se Cairomônios (Brown et al 1970; Whittaker \& Feeny, 1971).

Segundo Florkin (1966) e Margalef (1974), a manutenção de uma determinada comunidade biótica de um ecossistema não depende só dos fatores físicos e químicos do meio, mas também de muitas interaçōes químicas entre os organismos, as quais são muito importantes na diversificação dos ecossistemas e evolução das espécies.

Durante milhões de anos, os organismos desenvolveram um refinamento destas substâncias para funçōes diversas. A evoluçāo acidental de uma seqüência metabólica, resultando na produção de uma substância nociva para outros membros da comunidade daria uma vantagem seletiva para o clone que adquiriu esta capacidade. O decréscimo da predação sobre esses organismos, induziria um aumento da concentração destes compostos na natureza (Kittredge et al., 1974).

As saponinas esteroidais de Marthasterias glacialis (Echinodermata, Asteroidea) que desencadeiam reações de escape em moluscos, constituem exemplo de Cairomônios (Feder, 1967; Mackie, 1975) e todos os tipos de repelentes, inibidores, venenos e peçonhas são Alomônios.

Os venenos e peçonhas ocorrem em todos os Phyla do reino animal com exceção das aves (Russell, 1967) e estão intensamente estudados sob o ponto de vista toxicológico e farmacológico; entretanto, poucos esclarecimentos existem com relação ao papel fisiológico no organismo liberador, interaçōes na comunidade e evolução.

Segundo Freyvogel \& Perret (1973), no reino animal, as toxinas podem ser subdivididas em venenos e: peçonhas. Os venenos são produtos metabólicos, produzidos e acumulados em vários órgãos, que afetam quando ingeridos naturalmente ou quando aplicados artificialmente por via parenteral. Os organismos venenosos primários são aqueles que produzem metabolicamente os venenos como, por exemplo, o dinoflagelado tóxico, Gonyaulax catenella, produtor de uma potente neurotoxina que será discutida posteriormente. Os animais venenosos secundérios adquirem as toxinas através da cadeia trófica, alimentando-se de organismos venenosos primários, por exemplo, os mexilhōes podem adquirir a neurotoxina dos dinoflagelados tóxicos através da cadeia alimen- tar. Os mexilhões passam a ser "transvetores"; quando esses consumidores intermediários (bivalvos) forem ingeridos por animais de níveis tróficos superiores, o envenenamento poderá ocorrer (Sasner, 1973). As peçonhas são produzidas em glândulas especializadas e atuam parenteralmente até por simples contato; quando ingeridas, geralmente, são destruidas por enzimas digestivas. Nos Animais que só usam peçonhas para auto-defesa ("Venomous Defense Animals"), as estruturas traumagênicas são incompletas ou ausentes; p. ex. certos ouriços-do-mar (Asthenosoma varians) são dotados de glândulas peçonhentas nos espinhos. Finalmente, animais peçonhentos predadores ("Venomous Prey Animals"). São os que possuem estruturas traumagênicas especializadas, empregadas principalmente para a captura de presas, como por exemplo, os moluscos gastrópodes do gênero Conus, portadores de uma rádula dotada de arpões para a inoculação da peçonha.

Entre os invertebrados marinhos, compostos biologicamente ativos estão sendo encontrados em praticamente todos os grupos até agora estudados. Abordaremos a seguir apenas alguns grupos que estão sendo estudados em nossos laboratórios.

\section{Annelida}

Os anelídeos constituem um grupo com cerca de 8.800 espécies (Russell-Hunter, 1968) e a classe Polychaeta vem sendo muito pesquisada neste aspecto. $O$ emprego de Nereistoxina isolada do poliqueto Lumbriconereis heteropoda, como matéria prima para a fabricação do inseticida "CARTAP", constitui um bom exemplo do uso de uma substância biologicamente ativa de um animal marinho na indústria (Narahashi, 1973).

Outros produtos de poliquetos, como a Telepina, isolada de Thelepus setosus, possuem propriedades fungicidas (Higa \& Schewer, 1975) e os extratos de tentáculos de Lanice conchilega, do Hawaii, e Reteterebella queensland, da Australia, inibiram completamente o crescimento de tumores em camundongos (Tabrah et al., 1970). Nestes animais, os aparelhos defensivos ou para captura de presas são constituídos das cerdas dos parapódios, como é o caso de Eurythoë, ou as-mandíbulas, às vezes associadas à glândulas peçonhentas, como em Glycera (Halstead, 1959, 1965; Day, 1967; Southcott. 1970; Bücherl \& Bückley, 1971).

No litoral brasileiro, a especie circuntropical Eurythoe complanata (Fam. Amphinomidae) ocorre com certa freqüência em praias lodosas, sob rochas. Sawaya (1966) observou vários casos de pessoas que entraram em contato com esses poliquetos e que apresentaram graus diferentes de sensibilidade, provavelmente relacionados a reaçôes alérgicas. Yaldwyn (1965) observou que o contato com as cerdas deste animal provoca edema local, inchaços e que, após 15 horas, formam-se bolhas sanguíneas na região de penetração das cerdas. Vários autores afirmam que as cerdas são preenchidas com veneno (Halstead, 1965; Day, 1967; Baslow, 1969). Cada cerda é secretada por uma única célula do saco setal e existem músculos associados com as cerdas; os anelídeos podem, assim, retraílas ou extende-las quando perturbados. "Final determination of whether glandular elements are present or not awaits further research. Thus far, no one appears to have described any venom glands" (Bücherl \& Bückley, 1971). Estudos realizados com E. complanata evidenciaram que as cerdas são ocas e compostas de calcário e quitina. Extratos da parede do corpo, incluindo a região dos parapódios, contém um princípio ativo, dialisável, que provoca contração no íleo de cobaia e no músculo dorsal do próprio poliqueto e é antagonizada por antistina, antagonista da histamina; não sendo antagonizada por atropina e ácido $\alpha$-bromolisérgico, conhecidos antagonistas da acetilcolina e serotonina, respectivamente. 
Mollusca

Este grupo possui cerca de 110,000 espécies (Russell-Hunter, 1968), das quais oitenta e cinco estão envolvidas em envenenamentos humanos ou conhecidas como tóxicas em certas ocasiōes (Russell, 1971). Das classes existentes, três foram intensamente estudadas, Bivalvia, Cephalopoda e Gastropoda.

A Saxitoxina foi identificada como o composto biologicamente ativo adquirido pelos Bivalvos, através da cadeia alimentar. Segundo Twarog et al. (1972), possivelmente as espécies de bivalvos que são periodicamente expostas às marés vermelhas tóxicas, desenvolveram mecanismos que ihes permitiram coexistir com os dinoflagelados tóxicos. Entre as espécies estudadas, Mytilus edulis e $M$. californianus, a resistência à Saxitoxina não é conferida por uma bainha neural impermeável, nem depende do abandono de um mecanismo de $\mathrm{Na}$ + para geração do potencial de ação. Segundo os autores, um possível mecanismo seria uma "imunidade" específica do processo de condutância dos íons $\mathrm{Na}$ à toxina. B.C. Abbot (1978, comun. pessoal) falou de possíveis proteínas inativadoras da toxina no corpo dos mexilhões.

O merceneno, isolado do bivalvo Mercenaria mercenaria tem sido indicado como um composto biologicamente ativo com ação seletiva tóxica em tecidos tumorais (Grant \& Mackie, 1977).

Dos cefalópodes, principalmente das glândulas salivares posteriores dos polvos foram isoladas vários compostos biologicamente ativos, dentre os quais uma proteína, a Cefalotoxina, responsável pela paralisação das presas (Ghiretti, 1960); a Serotonina; a Tiramina e a Octopamina. Esta última amina vem sendo apontada como neurotransmissora, especialmente nos invertebrados, sendo que, nos mamíferos, parece ser co-mediadora da Noradrenalina (Axelrod \& Saavedra, 1977). Entre as quinze enzimas diferentes da saliva destes anmass, encontra-se a Hiahuronidase (Russell, 1971), que é considerada "fator de difusão" das peçonhas nos tecidos. O polvo australiano, Hapalochlaena maculosa, tem sido responsável por vários casos fatais; um mergulhador picado pelo animal, morre com parada respiratória em cerca de duas horas após o acidente. Esta toxina fatal, a Maculotoxina. é semelhante à Saxitoxina quanto à sua ação no sistema nervoso e nas membranas excitảveis, bloqueando o impulso nervoso por inibir a condutância do sódio, que normalmente ocorre após a estimulação.

Um polipeptideo de onze aminoácidos, Eledoisina, foi isolado das glândulas salivares de Eledone moschata (octopódo do Mar Mediterrâneo) e é 50 vezes mais potente na açāo hipotensora, quando comparado com Acetilcolina, Histamina e Bradicinina (Russell, 1971).

A pesquisa de compostos biologicamente ativos em moluscos gastrópodes iniciou-se com os trabalhos de Dubois (1903), demonstrando que extratos alcoólicos de Murex sp. provocam paralisia muscular, convulsōes e morte em răs e peixes. Mais tarde, Vicent \& Jullien (1938) demonstraram que os extratos destes animais provocam contraç̃es do músculo dorsal eserinizado da sanguessuga, concluindo que a ação é devida a um ou mais Colinésteres. Jullien (1940) demonstrou a presença nos extratos de apenas um Colinéster. Os trabalhos que se seguiram com estes materiais foram realizados por Erspamer et al. $(1948,1953)$, que identificaram Serotonina e Urocanilcolina e determinaram a fórmula molecular desta última.

Os estudos realizados com glândulas hipobranquiais revelaram, na família Thaididae, Urocanilcolina e Senecioilcolina e, na família Buccinidae, Acrililcolina (Whittaker, 1960). Em outras espécies, Thais chocolata e Choncholepas concholepas. Roseghini et al. (1970), isolaram Ürocanilcolina e Senecioilcolina e, mais tarde, Roseghini \& Fichman (1973), trabalhando com os mesmos órgãos de Thats haemastoma, encontraram predominantemente Dihidromurexina e Senecioilcolina, havendo muito pouca quantidade de Urocanilcolina, que pode, ocasionalmente estar ausente.

Segundo Endean (1972), Tetramina ocorre em glândulas salivares de algumas espécies de Buccinidae e Cymatidae e Huang \& Mir- (1972) encontraram uma toxina nas glândulas salivares de Thais haemastoma, tóxica para camundongos $\left(\mathrm{LD}_{50}=43 \mathrm{mg} / \mathrm{kg}\right)$ com açáo depressora no sistema nervoso central destes animais, forte ação hipotensora em gatos e ação estimulante intensa no íleo de cobaia e duodeno de coelho, não antagonizada por atropina ou hexametônio.

No gênero Conus, com aproximadamente 40 espécies, algumas espécies piscívoras são peçonhentas para o homem. $\mathrm{O}$ aparelho inoculador consiste de um bulbo muscular que armazena a peçonha secretada pelo longo duto peçonhento e um saco radular composto por dentes radulares em arpão que podem atingir até $10,9 \mathrm{~mm}$ em Conus striatus (Kohn et al., 1960). A peçonha contém vários componentes que provocam bloqueio neuromuscular, entre eles $\mathrm{N}-\mathrm{Me}$ tilperidina, Gamabutirobetaina e Homarina.

Com relação à família Aplysiidae, papéis defensivos têm sido atribuídos a três diferentes produtos quimicos destes gastrópodes (Stallard et al., 1974), o mais perceptivel é o fluído púrpura expelido por estes animais, quando perturbados. Estes autores atribujram a Kittredge et al. (1974) a informação de que, além de servir como nuvem protetora, a púrpura pode conter substâncias capazes de paralisar o aparelho quimiosensorial dos predadores. Uma outra secreçăo é o fluido branco leitoso proveniente da glândula de opalina (glândula de Bohadtch) situada na parte anterior da cavidade paleal, que é capaz de provocar paralisias e morte em vários organismos marinhos (Flury, 1915), O terceiro órgl̈o defensivo é o da glândula do intestino médio. estudado injeialmente em Aplysia call. fornica e $A$. naccuria por Winkler (1961) e Winkler et af. (1962), da qual isolaram um princípio colinomimético, cuja identificação química foi recentemente feita por Blankenship et al. (1975) como sendo a Urocanilcolina. Esta substância, já conhecida de outros moluscos, possui ação bloqueadora neuromuscular do tipo despolarizante (Quilliam, 1957), produz sintomas de hiper-estimulação e bloqueio dos receptores nicotínicos e tem reduzidos efeitos muscarínicos em vertebrados (Erspamer \& Glasser, 1957). Compostos orgânicos bromados estão presentes na parede do corpo e, em grande quantidade, na glândula do intestino médio, propiciando grande resistência aos predadores (Winkler, 1969).

Watson (1973) trabalhou com einco especies de aplisideos hawaianos: Dolabella auricularia, Aplysia pulmonica, Dolabrifera dolabrifera, Stylocheilus longicauda e A. juliana, empregando extrados de glândula digestiva, glândula de opalina, glândula da púrpura, do ovotestis, do estômago, intestino e papo. Determinou a letalidade em camundongos, por injeçăo intraperitonial e administração oral, além de alguns ensaios farmacológicos com o "peixeborboleta" hawaiano, Chaetodon miliaris. Somente um único caso de intoxicação por outro tecido diferente da glândula digestiva foi notado. $\mathrm{O}$ referente ao intestino de $A$. pulmonica. Em $A$. juliana nem mesmo o extrato da glandula do intestino médio foi tóxico. Watson, portanto, sugere que a toxicidade destes animais pode não ser necessariamente um fenômeno universal e, provavelmente, pode depender de variações na dieta, estação, local de coleta, e ainda, salienta: "if the aforementioned bromo compounds are indeed the toxic material (s), the lack of letality of $A$. juliane mid gut glands seems entirely logical because this particular species in known to subsist in Hawaii almost entirely upon' Ulva, (Kay, 1969) a macroscopic green alga which does not contain bromine". $\mathrm{O}$ autor ainda enfatiza que esses resultados poderiam também estar influenciados pelo grau de estabilidade e volatilidade da toxina, diferenças na eficiência de extração, degradação parcial pelos processos de extração etc. Posteriormente, Watson \& Rayner (1973) ensaiando extratos etéreos e aquosos em ratos, demonstraram que doses subletais do resíduo cru, soluv́el em água, injetadas por via intravenosa, produzem hipotensão transitória, bradicardia e apnéia e que a fraçāo solúveI em éter prođuz hipertensão não bloqueada por agentes antagonistas alfa e beta adrenérgicos e, ainda que o efeito hipotensivo não foi abolido por vagotomia ou tratamento prévio com atropina e benadril.

As relações entre a glândula đo intestino médio e a glândula de opalina, se é que existem, não são conhecidas (Watson, loc. cit.) e, segundo Blankenship et al. (1975), a similaridade do papel fisiológico da Urocanilcolina, tanto em prosobrânquios como,opistobrânquios, poderia ficar estabelecida se fosse comprovada a sua presença tanto na glândula da púrpura como na de opalina destes moluscos. $\AA$ vista desses resultados o autor do presente trabalho empreendeu estudos farmacológicos com extratos da glândula do intestino médio de Aplysia brasiliana, uma das espécies freqüentes no litoral brasileiro. Demonstraram que esses extratos contêm princípio (s) colinomimético (s). Uma das ações deste (s) princípio (s) foi uma surpreendente interrupção da ação vagal sobre o coração do sapo. Como se sabe, a ação da Acetilcolina liberada pelo nervo vago no coração pode ser interrompida por meio de drogas ganglioplégicas despolarizantes sintéticas, como o Hexametônio ou Tetraetilamônio que agem ao nível das sinapses intramurais. A Urocanilcolina talvez presente nos extratos, também tem ação despo. larizante e, daí, pode ser que no coração do sapo este Colinéster tenha despolarizado mais persistentemente a região do "pacemaker", tornando inócua a ação da Acetilcolina liberada pelo nervo vago (Freitas, 1976). Com o emprego da metodologia adotada por Blankenship et al. (loc. cit.) e de outros ensaios farmacológicos com estruturas colinoceptivas, comprovou-se a presença de três Colinésteres, tanto na glândula do intestino médio, como na de opalina e dois na glândula da púrpura (Freitas, 1977). As pesquisas em andamento com. $\boldsymbol{A}$. juliana também parecem indicar a presença de Colinésteres na glândula do intestino médio. 
Arthropoda

Halstead (1965) enumerou oito espécies de artropodes marinhos considerados tóxicos: três da família Xiphosuridae (Carci. noscorpus rotundicauda, Tachypleus gigas e $T$. tridentatus), uma da fam. Sacculinidae (Sacculina carcini), um da fam. Hippidae (Emerita analoga) e três da fam. Xanthidae (Angatea sp, Eriphia norfolcensis, Xanthodes reynaudi). As intoxicaçōes humanas pelo xiphosurídeos, pelo menos para a espécie Carcinoscorpius rotundicauda, parecem ser devidas a um componente tóxico das massas de ovos, tecidos e víscezas durante a estação reprodutiva do ano, que usualmente dentro de trinta minutos provoca tonturas, dores de cabeça, náuseas, diminuicão do pulso, vômitos e fraqueza geral (Trishnananda et al., 1966). Outros sintomas mais severos podem ocorrer como paralisias musculares, perda da consciência e a morte sobrevem em 16 horas. O cirripédio $S$. carcini é uma parasita dos tecidos de caranguejos, que parece elaborar substâncias desconhecidas quimicamente, inibidoras do crescimento do seu hospedeiro e alterar suas características sexuais (Lévy, 1923; Halstead, 1965). Em relação aos anomuros, $E$. analoga possui uma toxina que tem sido registrada como a Saxitoxina, obtida provavelmente pela ingestão de dinoflagelados do gênero Gonyaulax, que produz paralisia, coma e morte, quando injetada intraperitonialmente em camundongos (Sommer, 1932). Bagnis (1970) registrou um caso clínico de envenenamento por "coconut crab" (Birgus latro), um anomuro terrestre amplamente distribuído em certos atóis do Pacífico, que provoca os seguintes sintomas: hipersecreção, tontura, ileo adinâmico e adinamia.

Em relação aos crustáceos decápodes braquiuros, foi realizada uma investigação epidemiológica nas llhas de Ryukyu e Amami, no Japão, e as espécies Platypodia granulosa e Zosimus aeneus provoçaram intoxicaçōes com sintomas e sinais semelhantes aos induzidos por baiacus e mariscos toxicos, ou seja, envenenamento por tetrodotoxina e saxitoxina, respectivamente. $\mathrm{O}$ produto dos caranguejos foi facilmente dializável, solúvel em água e metanol e insolúvel em muitos solventes orgânicos (Hashimoto et al., 1967, 1969). Mais tarde Konosu et al. (1968) estudaram a "crab toxin" comparativamente com a saxitoxina e a tetrodotoxina, tendo em vista a grande semelhança farmacológica destes compostos. A análise cromatográfica em papel e camada delgada da "crab toxin", obtida do exoesqueleto de Zosimus aeneus, mostrou semelhança com a saxitoxina e acentuadas diferencas com a tetrodotoxina.

Inoue et al. (1968) examinaram 722 espécimes, abrangendo 56 espécies de várias illas japonesas, e encontram um novo caranguejo tóxico, o Atergatis floridus. A toxicidade de três espécies de caranguejos xantídeos foi estudada por Konosu et al. (1969), os quais examinaram 145 espécies de $Z$. aeneus, 35 de $P$. granulo sa e 144 de $A$. floridus, coletadas nas Ithas Amami e Oshima, em todas as estaçōes anuais. Estes autores determinaram a quantidade de toxina em diferentes tecidos em $Z$. aeneus, espécie considerad muito tox́ica e, em ordem decrescente de toxidez, o resultado desta análise foi o seguinte: exoesqueleto e músculos dos apêndices; visceras; músculos do cefalotórax, endofragma e brânquias e, finalmente, os ovários. Segundo eles, existem registros de intoxicaçōes humanas por caranguejos tóxicos no Arquipélago Fiji e nas Ilhas Gilbert, estendendo-se assim, a distribuição dos mesmos, ao sul do Oceano Pacífico.

Noguchi et al. (1969) identificaram a "crab toxin" como sendo a própria Saxitoxina, empregando a cromatografia de trocaiônica e espectro de absorção infra-vermelho. Entretanto, Teh \& Gardiner (1970) distinguiram a "crab toxin" das conhecidas neurotoxinas, Saxitoxina e Tetrodoxina, baseando-se em certas propriedades químicas e farmacológicas.

Posteriormente, duas outras espécies foram registradas como tóxicas nas Filipinas, Demania toxica e Lophozozymus pictor (Garth, 1972; Alcala \& Halstead, 1970; Teh \& Gardiner, 1970, 1974; Gonzalez \& Alacala, 1977).

Iim interessante aspecto comportamental foi notado pelo Autor deste trabalno em alguns crustáceos decápodes braquiuros, durante coletas no litoral do Estado de São Paulo (Brasil). Espécies, tais como: Eriphia gonagra, Pachygrapsus transversus, Goniopsis cruentata, Mithrax hispidus. Quando manuseados e expostos a variações térmicas, esses animais expelem, através da boca, seu suco digestivo, que juntamente com a água bombeada da câmara branquial, forma uma espuma que chega a cobrir a carapaça, sugerindo um comportamento defensivo. Sabe-se que vários insetos, como ortópteros, mantódeos, larvas de lepidópteros frequentemente regurgitam uma gota de fluido intestinal através da boca, quando são manuseados e em alguns casos o fluido tem tunção repelente contra predadores (Brower \& Brower, 1964). Ensaios farmacologicos preliminares com os fluidos digestivos e homogeneizados de glândulas do intestino médio dos crustáceos menciona- dos sugerem a presença de um (ou mais) composto biologicamente ativo solúvel em água, termo-estável e dializável que diminui a excitabilidade muscular de forma reversível. Esta pesquisa se acha em andamento no sentido da melhor purifiçação da (s) substância (s), a fim de esclarecer ações farmacológicas e interações entre os organismos na comunidade.

\section{Conclusర̃es}

Os estudos no campo dos compostos biologicamente ativos de origem marinha ainda são escassos na América Latina, talvez pela muito maior atenção ainda voltada para a agncultura e a pecuária, em detrimento do interesse pelos recursos marinhos. Todavia estudos de ecologia química, face a perspectiva de uma maior utilização desses recursos deveriam ser deste já realizados, com vistas a um crescente e mais variado consumo de organismos marinhos, envolvendo um maior contato com o mar por parte dos exploradores desses recursos. Recorde-se que, nesse contexto, tornou-se necessário recentemente fabricar soros anti-venenos contra medusas (Baxter et al., 1968). Além de se constituirem num inestimável potencial de fontes de proteínas, os organismos marinhos vêm formecendo, pelos compostos bioativos que possuem, importantes ferramentas para pesquisa fisiológica e, até, matéria prima para defensivos agrícolas, como é o caso da Nereistoxina. Drogas com finalidades terapêuticas, como os nucleosídeos arabinosínicos, isolados de esponjas (Bergmann \& Feeney, 1951) têm servido como matéria prima para inibidores de tumores e o Ácido Kaínico isolado da alga rodofícea Digenea simplex é usado como anti-helmíntico especialmente no Japão (Murakami et al., 1955).

Certas espécies marinhas, ainda necessitam de melhores investigações, como é o caso, p. ex., do gênero Aplysia. Kandel (1976) salienta que os estudos neurofisiológicos deste animal se acham muito bem adiantados, tendo a espécie sido selecionada pelos neurobiologistas devido às grandes vantagens de análise de seu sistema nervoso central, cujos neurônios gigantes servem como bons materiais para pesquisa. Mas, segundo o autor, muito pouco foi realizado sobre a fisiologia dos outros sistemas e o comportamento.

Muitos aspectos químicos, toxicológicos, farmacológicos e as implicações médicas de compostos biologicamente ativos de invertebrados marinhos estão muito bem detalhados nos trabathos de Courville et al. (1958); Halstead (1965); Russell (1965), 1967, 1971); Russell \& Saunders (1967); Nigrelli et al. (1967) Southcott (1970); Baslow $(1969,1971)$; Bücherl \& Buckley (1971); Mebs (1973); Martin \& Padilha (1973); Ruggieri (1976) e Scheuer (1964, 1977).

Para finalizar, quero apresentar parte do editorial apresentado por Halstead (1976) a The American Academy of Clinical Toxicology and American Association of Poison Control Center Joint Meeting: “... a Saxitoxina está em terceiro lugar entre os mais potentes venenos, apresentando um MLD em torno de 9 $\mu \mathrm{g} / \mathrm{kg}$; assim, e superada apenas pela toxina botulınica $\mathrm{A}$ e pela neurotoxina de Cobra. Sem dúvida. é cerca de 3.000 vezes mais toxica do que o Cianeto de Sódio - o veneno de escolha "for dispatching" agentes da CIA, KGB, and FBI". ..." As poucas gramas de Saxitoxina agora nas mãos da CIA, representam uma despesa de vários milhões de dólares, as carreiras de notáveis homens de ciêncie e muitas décadas. As biotoxinas envolvidas nas investigações da CIA são de extremo valor científico e muito importantes para esclarecimentos de neuroquímica e neurofisiologia básicas. As atividades biodinâmicas destas toxinas são profundas e da maior importância em nossas investigaçōes e na elucidação dos processos patológicos humanos: "someday, it will be seen that these noxious biotoxinas possess a dual role of being able to preserve life as well as destroy it"

\section{Agradecimentos}

Sou muito grato ao Prof. Dr. E.G. Mendes pela troca de idéias e revisão do manuscrito e ao Prof. Dr. Edmundo Nonato pela identificação da espécie Eurythoë complanata.

\section{Bibliografia}

ALCALA, A.G. \& HALSTEAD, B.W. 1970 Human fatality due to ingestion of the crab Demania $s p$ in the Philippines. Clinical Toxicol., 3 (4): 609-611.

AXELROD, J. \& SAAVEDRA, J.M. 1977 Octopamine. Nature, 265:501.

BAGNIS, R. 1970 A case of coconut crab poisoning. Clinical Toxicol., 3(4): 585-588.

BASLOW, M.H. 1960 Marine pharmacology. A study of toxins and other biologically active substances of marine origin. Baltimore, Williams \& Wilkins, 286p. 
BASLOW, M.H. 1971 Marine toxins. Ann. Rev. Pharmacol. 11: $447-454$.

BAXTER, E.H.: MARR, A.G. \& LANE, W.R. 1968 Immunity to the venon of the sea wasp, Chironex flexkeri. Toxicon, 6 45-50.

BERGMANN, W. \& FEENEY, R.J. 1951 Contributions to the study of marine products. XXXII. The Nucleosides of sponges. I.J. Org. Chem., 16:981-987.

BLANKENSHIP, J.E.; LANGLAIS, P.J. \& KITTREDGE, J.S. 1975 Identification of a cholinomimetic compound in the digestive gland of Aplysia californica. Comp. Biochem. Physiol,, SIC: $129-137$

BROWER, L.P. \& BROWER J.V.Z. 1964 Birds, butterflies, and plant poisons: a study in ecological chemistry. Zoologica, N.Y., 49:137-159.

BROWN Jr., W.L.; EISNER, T. \& WHITTAKER, R.H. 1970 Allomones and kairomones: transpecific chemical messengers. Bioscience, 20:21.

BUCHERL. W. \& BUCKLEY. E.E. 1971 Venomous animals and their venoms. v. 3 Venomous invertebrates. New York, Academic Press, 537p.

COURVILLE, D.A.; HALSTEAD, B.W. \& HESSEL, D.W. 1958 Marine biotoxins: isolation and properties. Chem. Rev., 58: $235-248$.

DAY, J.H. 1967 Polychaeta of Southern Africa, Part I. Errantia. London. Brit. Mus. (Nat. Hist.), 458p.

DUBOIS, R. 1903 Sur le venin de la glande à pourpre des Murex. Apud RUSSELL, F.E. 1971.

ENDEAN, R. 1972 Aspects of molluscan pharmacology. In: FLORKIN, M. \& SCHEER, N.Y., ed. Chemical żoology. Mollusca, 7. New York, Academic Press. p. 421-466.

ERSPAMER, V. 1948 Observazioni preliminari chimiche e farmacologiche, sulla murexina. Experientia, 4:226-228.

ERSPAMER, V. \&BENATI, O. 1953 Identification of murexine as beta (imidazolyl-(4)-acryl-choline. Science, 117 161-162.

ERSPAMER, V. \& GLASSER, A. 1957 The pharmacological actions of murexina (Urocanylcholine). Brit. J. pharmacol. Chemother., 12:176-184.

FEDER, H.M. 1967 Apud MACKIE, A.M. 1975.

FLORKIN, M. 1966 Aspects moleculaires de l'adaptation et de la phylogénie. Paris, Masson, 258p.

FLURY, F. 1915 Uber das Aplysiengift. Apud RUSSELL, F.E 1971.

FOX, H.M. 1924 Apud MACKIE, A.M. 1975

FREITAS, J.C. 1977 Ocorrência de esteres de cotuna em Aplysia brasiliana Rang, 1828 (Mollusca, Gastropoda, Opisthobranchia). 2? SEMANA DE SEMINÁRIOS DE BIOLOGIA MARINHA, São Sebastião, S.P. - Anais Acad. bras. Ciênc. (No prelo).

FREY VOGEL, T.A. \& PERRET, B.A. 1973 Notes on toxinology. Experientia, $29(11): 1317-1452$.

GARTH, J.S. 1972 Demania toxica, a new species of poisonous crab from the Philippines. Micronesica, $7(1 / 2): 179-183$.

GHIRETTI, F. 1960 Toxicity of octopus saliva against Crustacea. Ann. N.Y. Acad. Sci., 90:726-741.

GONZALEZ, R.B. \& ALCALA, A.C. 1977 Fatalities from crab poisoning on Negros Island, Philippines. Toxicon, 15:169. 170.

GRANT, P.T. \& MACKIE, A.M. 1977 Drugs from the sea: fact or fantasy? Nature, 267: 786-788

HALSTEAD, B.W. 1959 Dangerous marine animals. Cambridge, Cornell marit. Press. $146 \mathrm{p}$.

HALSTEAD, B.W. 1965 Poisonous and venomous marine animals of the world. I. Invertebrates. Washington, U. S. Gov. Printing Office, $994 \mathrm{p}$.

HALSTEAD, B.W. 1976 Shellfish emotionalism. Clinical Toxico$\log y .9(1):-1-2$.

HASHIMOTO, Y.; KONOSU, S.; INOUE, A. \& NOGUCHI, T. 1967 Ocurrence of toxic crabs in Ryukyu and Amami Islands. Toxicon, 5: 85-90.

HASHIMOTO, Y.; KONOSU, S.; INOUE, A.; SAISHO, T. \& MIYAKE, S. 1969 Screening of toxic crabs in the Ryukyu and Amami lslands. Bull. Jap. Soc. scient. Fish., 35 (1): 83-87.

HIGA, T. \& SCHEWER, P.T. 1975 Antitumor activity in mice of tentacles of two tropical sea annelids. Apud GRANT, PIT. \& MACKIE, A.M. 1977.

HOWE, N.R. \& SHEIKH, Y.M. 1975 Anthopleurine. A sea-anemone alarm pheromone. Science, 189:386-388.

HUANG, C.L. \& MIR, G.N. 1972 Pharmacological investigation of salivary gland of Thais haemastoma (Clench). Toxicon, 10:111-117.

INOUE. A.; NOGUCHI. T.; KONOSU. S. \&HASHIMOTO. Y. 1968 A new toxic crab, Atergatis floridus. Toxicon, $6: 119-123$
JULLIEN, A. 1940 Variations dans les temps de la teneur des extraits de glande à pourpre en substances actives sur le muscle de sangsue. C.r. Séanc. Soc. Biol., 123: 524-527.

KANDEL, E.R. 1976 Cellular basis of behaviour. An introduction to behavioral neurobiology. San Francisco, W.H. Freeman, $727 \mathrm{p}$.

KARLSON, P. \& LUSCHER, M. 1959 Pheromones: a new term for a class of biologically active substances. Nature, Lond., 183: 55-56.

KAY, E.A. 1969 The aplysiidae of the Hawaiian Islands. Apud WATSON, M. 1973.

KITTREDGE, J.S.; TAKAHASHI, F.T.; LINDSEY, J. \& LASKER, R. 1974 Chemical signals in the sea: marine allelochemics and evolution. Bull. Fish. Wildl. Serv. U.S., 72: 1-12.

KOHN, A.J.; SAUNDERS, P.R. \& WIENER' S. 1960 Preliminary studies on the venom of the marine snail Conus. Ann. N.Y. Acad. Sci., 90:706-725.

KONOSU, S.; INOUE, A.; NOGUCHI, T. \& HASHIMOTO, Y. 1968 Comparison of crab toxin with Saxitoxin and Tetrodotoxin. Toxicon, 6:113-117.

KONOSU. S. INOUE. A.: NOGUCHL. T. \& HASHIMOTO Y. 1969 A further examination on the toxicity of three species of xanthid crab. Bull. Jap. Soc. scient. Fish., 35 (1): 88-92.

LÉVY, R. 1923 Sur la toxicité des tissus de la sacculine (Sacculina carcini Thomp.) vis-à-vis du crabe (Carcinus maenas Penn.) et sür la recherche de réactions d'immunité chez ce dernier. Bull. Soc. Zool. Fr., 48: 291-294.

MACKIE, A.M., 1975. Chemoreception. In: MALINS, D.C. \& SARGENT, J.R., ed. - Biochemical and biophysical perspectr ves in marine biology, II. New York, Academic Press, 359p.

MACKIE, A.M. \& GRANT, P.T. 1974 Interspecies and intraspecies chemoreception by marine invertebrates. In: GRANT, P.T. \& MACKIE, A.M., Chemoreception in marine organisms. London. Academic Press, 295p.

MARGALEF, F., 1974. Ecologia. Barcelona, Ediciones Omega, $951 \mathrm{p}$.

MARTIN, D.F.\& PADILLA, G.M., 1973 Marine pharmacognosy. Action of marine biotoxins at the cellular level. New York. Academic Press, 317p.

MURAKAMI, S.; TAKEMOTO, T.; TEI, Z. \& DAIGO, K. 1955 Effective principles of Digenea simples VIII. Structure of kainic acid. Apud MARTIN, D.F. \& PADILLA, G.M., ed. 1973.

NARAHASHI, T. 1973 Mode of action of Nereistoxin on excitable tissues. In: MARTIN, D.F. \& PADILLA, E.M., ed. 1973.

NIGRELLI, R.F. STEMPIEN, Jr. M.F.; RUGGIERI, G.D.; LIGUORI, V.R. \& CECIL, J.T. 1967 Substances of potencial biomedical importance from organisms. Federation Proc., 26 (4): 1197-1205.

NOGUCHI, T; KONOSU, S. \& HASHIMOTO, Y. 1969. Identity of the crab toxin with Saxitoxin. Toxicon, 7:323-326.

QUILLIAM, J.P. 1957 The mechanism of action of Murexine on neuromuscular transmission in the frog. Br. J. pharmac. Chemother., 12:388-392.

RASKOVÁ, H., ed. 1971 Pharmacology and toxicology of naturally occurring toxins. IUPHAR, v.2. Oxford, Pergamon Press. 299v.

ROSEGHINI, M; ERSPAMER, V.; RAMORINO, M.L. \& GUTIERREZ, J.E. 1970 Choline esters, their precursors and metabolites in the hypobranchial gland of prosobranchiate moltuscs, Concholepas concholepas and Thais chocolata. Eur. J. Biochem., 12:468-473.

ROSEGHINI, M. \& FICHMAN, M. 1973 Choline esters and imidazole acids in extracts of the hypobranchial gland of Thais haemastoma. Comp. gen. Pharmacol., 4: 251-257.

RUGGIERI, G.D., 1976 Drugs from the sea. Marine organisms with novel chemical constituents are excellent sources of new idrugs. Science, 194: 491-497.

RUSSELL, F.E., 1965. Marine toxins and venomous and poisonous marine animals. Adv. mar. Biol., 3: 284 p.

RUSSELL, F.E. 1967 Comparative pharmacology of some animal toxins. Federation Proc., 26 (4): 1206-1224.

RUSSELL, F.E. 1971 Pharmacology of toxins of marine organisms. In: RASKOVÃ, H. ed. 1971

RUSSELL-HUNTER. W.D. 1968 A biology of lower invertebrates. New York, MacMillan, $181 \mathrm{p}$.

RUSSELL, F.E. \& SAUNDERS, P.R. 1967 Animal toxins. Oxford, Pergamon Press, 428p.

SASNER, Jr., J.J., 1973 Comparative studies on algal toxins. In: MARTIN, D.F. \& PADILLA, G.M., ed. 1973.

SAWAYA, P.. 1966 Toxic marine invertebrates. Venomous and noxious fisches of fresh water. Mem. Inst. Butantan, $33(1): 31-34$. 
SCHEUER, P.J. 1964 The chemistry of toxins isolated from some marine organisms. Forthschr. Chem. Org. NatStoff, 22: 265-278.

SCHEUER, P.J. 1977 Chemical communication of marine invertebrates. BioScience, 27 (10): 664-668.

SOMMER, H., 1932. The occurrence of the paralytic shellfish poison in the common sand crab. Science, 76:574-575.

SONDHEIMER, E. \& SIMEONE, J.B. 1970 Chemical ecology. Apud KITTREDGE, J.S.; TAKAHASHI, F.T.; LINDSEY, J. \& LASKER, R. 1974.

SOUTHCOTT, R.V., 1970. Human injuries from invertebrate animals in the Australian Seas. Clinical Toxicol, 3 (4) 617-636.

STALLARD, M.O. \& FAULKNER, D.J., 1974. Chemical constituents of the digestive gland of the sea hare Aplysia cali. fornica. - I. Importance of diet. Comp. Biochem. Physiol., $49 B: 25-35$

TABRAH, F.L.; KASHIWAGI, M. \& NORTON, T.R. 1970 Antitumor activity in mice of tentacles of two tropical sea annelids. Science, 170:181-183.

THRISHNANANDA, M.; TUCHINDA, D.; YIPINSOI, T. \& OONSOMBAT. P. 1966 Poisoning following the ingestion of the horseshoe crab (Carcinoscorpius rotundicauda): Report of four cases in Thailand. Apud BASLOW, M.H. 1960.

TWAROG, B.M.; HIDAKA, T. \& YAMAGUCHI, H. 1972 Resistance to Tetrodotoxin and Saxitoxin in nerves of bivalve molluscs. A possible correlation with paralytic shellfish poisoning. Toxicon, 10:273-278.

VICENTE, D. \& JULLIEN, A. 1938 Richesse de la glande a pourpre des Murex en esters de la choline, C.r. Séanc. Soc. Biol. 127: 1506 .

WATSON, M., 1973. Midgut gland toxins of Hawaiian sea hares. I. Isolation and preliminary toxicological observation. Toxicon, $11: 259-267$.

WATSON, M. \& RAYNER, M.D. 1973 Midgut gland toxins of Hawaiian sea hares. II. A preliminary pharmacological study. Toxicon. 11:269-276.

WHITTAKER, V.P., 1960 Pharmacologically active choline esters in marine gastropods. Ann. N.Y. Acad. Sci., 90:695-705.

WHITTAKER, R.H. \& FEENY, P.P. 1971 Allelochemics: chemical interaction between species. Science, 171:757-770.

WINKLER, L.R. 1961 Preliminary tests of the toxin extracted from Californian sea hares, of the genus Aplysia. Pacific Sc., 15 211-214.

WINKLER, L.R. 1969 Distribution of organic bromine compounds in Aplysia californica Cooper, 1963. Veliger, 11:268-271

WINKLER, L.R.; TILTON, B.E. \& HARDINGE, M.G. 1962 A cholinergic agent extracted from sea hares, Arch. Int. Pharmacodyn., $137(1 / 2): 76-83$.

YALDWYN, J.C. 1965. Blistering from bristle worm. Aust. Nat. Hist., 15: 86. 